\title{
Power Supply Optimization for the Superconducting Coil System of HELIAS Fusion Reactor
}

\author{
O.Gaupp, R.Haller, E.Harmeyer, J.Mühlbacher, A.Wieczorek, H.Wobig
}

\begin{abstract}
A power supply system for feeding the superconducting coils of the Helias reactor, an upgraded system of Wendelstein $7 \mathrm{X}$, has been investigated. These investigations on operation of the power supply of the fusion power plant were made using the SIMPLORER, EFFI and NEPLAN codes. This multiconverter supply system has been optimized, in view of low losses in the components and only little negative impact to the power grid. The design of the optimized multiconverter supply system was studied by means of computer simulations. The influence of the passive structures on operation of the power supply system was taken into account. The computation of induced eddy currents in the coil structure during transient processes are transformed into electric network analyses using the inductance and resistance data of the nonplanar coils and their coil housings, by means of the Finite Element Network (FEN) method. This approximation allows the investigation of the whole coil system including power supplies and passive structures. The new approach was to use an additional 80MW, 1.2GJ energy storage unit with the magnetic confinement system as a SMES in order to reduce network loadings to $80 \mathrm{MW}$ instead of $160 \mathrm{MW}$ in the power plant start-up phase during plasma heating. The reduction of the active power pulse loads reduces the risk of oscillating power frequency changes. These oscillations produce transient power flows which can danger the grid stability.
\end{abstract}

Index Terms - Fusion reactors, Reactive power, Power quality, Power supplies, Power system harmonics, Simulation, Superconducting magnets

Manuscript received October xx, 2008

O.Gaupp is with the ABB, Dept. Advanced Power Electronics, CH-5300 Turgi, Switzerland (e-mail: osvin.gaupp@ch.abb.com)

R.Haller is with the Pilsen University in Pilsen, Czech Republik (e-mail: rhaller@kee.zcu.cz)

E.Harmeyer was with the Max-Planck-Institut für Plasmaphysik (IPP), D85748 Garching, Germany (e-mail: ebh@ipp.mpg.de).

J.Mühlbaher is with the Pilsen University in Pilsen, Czech Republik (email:muhl@kee.zcu.cz)

A.Wieczorek is with the University of Applied Sciences (Fachhochschule) Regensburg, D-93053 Regensburg, Germany (e-mail: andreas.wieczorek@etechnik.fh-regensburg.de).

H.Wobig was with the Max-Planck-Institut für Plasmaphysik (IPP), D85748 Garching, Germany (e-mail: hwg@ipp.mpg.de).

\section{INTRODUCTION}

$\mathrm{T}$ he goal of fusion research is to derive energy from fusion of light atomic nuclei. In the process a helium nucleus is produced, this being accompanied by release of a neutron and large quantities of useful energy (Figure 1) [1], [2]. The raw materials needed to operate a fusion reactor; deuterium and lithium are available in practically unlimited quantities uniformly distributed throughout the world. Seawater contains deuterium in almost inexhaustible quantities. Tritium, a radioactive gas, hardly occurs in nature. It can, however, be formed in a power plant from lithium, which is likewise abundantly available. Since, moreover, a fusion power plant will have ecologically favorable properties, fusion could make an enduring contribution to future energy supply [3]-[5]. The successful development of nuclear fusion for generating power should provide an almost limitless source of energy for future generations.

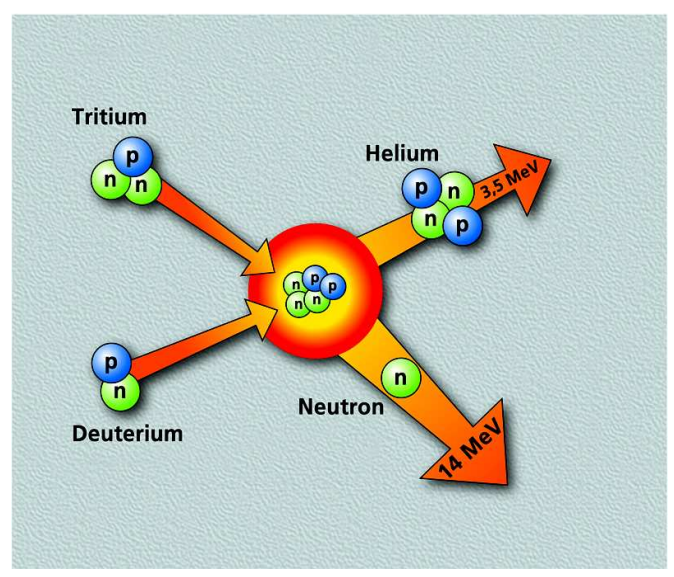

Fig. 1. Nuclear fusion reactions [1], [2].

The Stellarator System Studies group at Max-PlanckInstitut für Plasmaphysik (IPP) in Garching and Greifswald is concerned with investigating the physical and technological issues underlying a nuclear fusion power plant based on the stellarator concept. For this reason the HELIAS (HELIcal Advanced Stellarator) configurations have been developed. HELIAS configurations offer an optimized magnetic confinement concept for the HeliaS Reactor (HSR) [3]-[5]. 


\section{HELIAS REACTOR}

The stellarator as a steady-state fusion power plant offers several advantages compared to tokamak reactors:

- The inherent potential of steady state operation,

- The absence of a toroidal plasma current,

- The absence of plasma disruptions in stellarator configurations.

The HELIAS configuration is one of the favourite candidates for a steady state fusion reactor. This configuration has been developed at the IPP Garching in a long phase of analytic and numerical studies, which aim to improve the reactor prospects of a classical stellarator.

Two reactor versions have been investigated, a five periodic arrangement HSR5/22 with $22 \mathrm{~m}$ and a four periodic system HSR $4 / 18$ with $18 \mathrm{~m}$ major radius. The fusion output is in the range of $3 \mathrm{GW}$. The magnetic field on axis is chosen to be 4.75T in the first version, and to be $5 \mathrm{~T}$ in the second one, resp., resulting in a total stored magnetic energy in the coil system of about $100 \mathrm{GJ}$ in both cases.

TABLE I

MAIN PARAMETERS OF HSR4/18 AND HSR5/22

\begin{tabular}{lll}
\hline \hline & HSR4/18 & HSR5/22 \\
Major radius [m] & 18 & 22 \\
Av.minor radius [m] & 2.1 & 1.8 \\
Plasma volume [m ${ }^{3}$ ] & 1567 & 1407 \\
$\begin{array}{l}\text { Av.magnetic field on } \\
\text { axis [T] }\end{array}$ & 5.0 & 4.75 \\
$\begin{array}{l}\text { Max.magnetic field on } \\
\text { coils [T] }\end{array}$ & 10.3 & 10.0 \\
$\begin{array}{l}\text { Number of coils } \\
\text { Magnetic energy [GJ] }\end{array}$ & 40 & 50 \\
\hline \hline
\end{tabular}

Recent studies have been focused on a 4-period Helias configuration HSR4/18, which comprises 40 modular coils in total, and 10 coils per toroidal field period and is a more compact option for an ignition experiment than the five period configuration HSR 5/22, which may lead to a $20 \%$ cost reduction of the reactor core.

\section{COIL SYSTEM OF THE Helias Reactor}

Owing to its high temperature fusion plasma, the "fourth aggregate state of matter", cannot be confined direct in material vessels. Any wall contact would immediately recool the thin gas. Using magnetic fields, which confine and thermally insulate the fuel, keeping it away from the vessel walls, obviates the problem.

Charged particles - ions and electrons - are forced in a magnetic field into circular and helical orbits around the magnetic field lines. The particles are thus tied to the field lines. In a suitably shaped magnetic field cage it is therefore possible to confine plasma and keep it away from material walls

In a Helias reactor there is only one coil system generating the confining magnetic field. This makes stellarators suitable for continuous operation, whereas tokamaks without auxiliary facilities operate in pulsed mode.

The main technical component of the HELIAS reactor is the modular coil system [6]. Because of the well-established industrial data base and the good feasibility, niobium-titanium (NbTi) is foreseen as superconducting material for this the proposed 'cable-in-conduit' conductor. The superconductor is embedded in an $\mathrm{Al}$ jacket. Superfluid helium as coolant at an operating temperature of $1.8 \mathrm{~K}$ is necessary, because of the maximum field of $11 \mathrm{~T}$ at coil. For the proposed system with a $\mathrm{NbTi}$ superconductor at $1.8 \mathrm{~K}$, two cooling systems are required; a system of supercritical Helium at $4.2 \mathrm{~K}$ for cooling of the coil housings and the support structure, and a system of superfluid Helium at $1.8 \mathrm{~K}$ for cooling of the windings. This magnetic confinement system of the HSR $4 / 18$ consists of 40 nonplanar coils, modularely arranged in 4 toroidal periods with 10 coils per period (Figure 2.). The magnetic energy of the coil system is a rough figure of merit for the costs of the coil system.

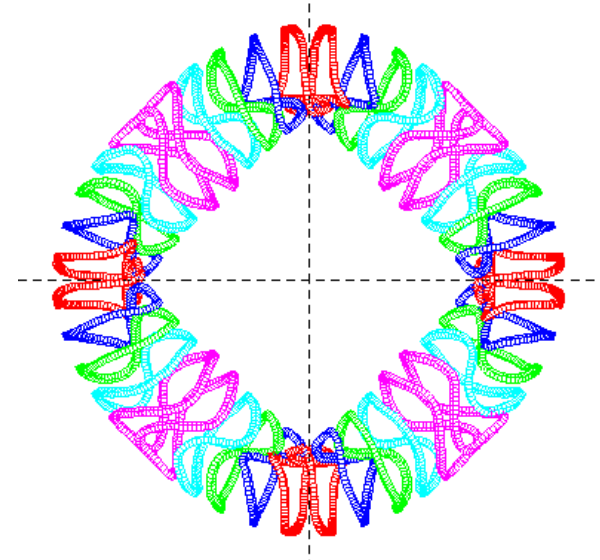

Fig. 2. The magnetic confinement system of the HSR4/18

As an alternative to this proposal other superconductors are currently investigated. Particularly, niobium-aluminum $\left(\mathrm{Nb}_{3} \mathrm{Al}\right)$ seems to have the potential for future application in large coil systems. This advanced approach offers higher magnetic fields than $\mathrm{NbTi}$, and a coolant temperature of $4 \mathrm{~K}$ could be used. Furthermore, $\mathrm{Nb}_{3} \mathrm{Al}$ withstands better mechanical stresses and is less sensitive under strain than $\mathrm{Nb}_{3} \mathrm{Sn}$.

In order to withstand the large magnetic forces in the modular nonplanar coils, the winding pack will

be surrounded by a coil housing of stainless steel. The coil winding pack is split into 8 double-pancakes, using a trapezoidally shaped cross-section and containing a total number of $18 \times 2 \times 8=288$ turns per coil (Figure 3 ). 


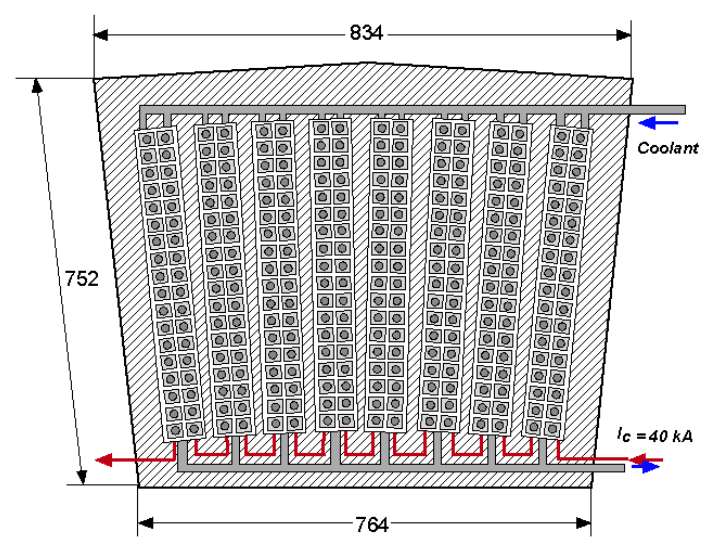

Fig. 3. Cross section of a coil surrounded by a stainless steel housing

The coil system is enclosed in a cryostat of roughly $16 \times 16$ $\mathrm{m}^{2}$ cross section (Figure 4.). Volume of the cryostat in $\mathrm{HSR} 4 / 18$ is $21500 \mathrm{~m}^{3}$.

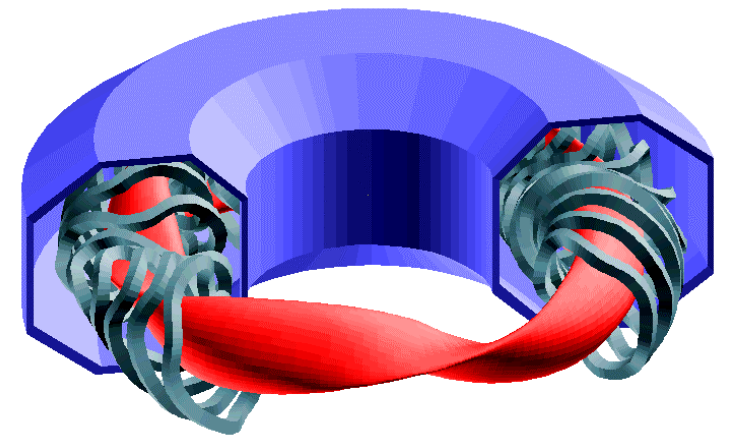

Fig. 4. Magnetic surface, modular coils and cryostat of the 4-period HELIAS reactor HSR4/18.

\section{POWER SUPPLY SYSTEM}

The electrical circuit of the coil system comprises 5 superconducting coil groups, each consisting of 8 equallyshaped coils which are electrically connected in series. These coil groups are magnetically coupled by the toroidal mutual reactance. The symmetrical inductance matrix $\mathbf{L}_{\text {HELIAS }}$ (in $\mathrm{H}$ )

$$
\mathbf{L}_{\text {HELIAS }}=\left[\begin{array}{ccccc}
17.64 & 4.84 & 1.92 & 0.80 & 0.74 \\
& 14.28 & 3.73 & 1.37 & 0.95 \\
& & 13.42 & 3.23 & 1.70 \\
& & & 13.06 & 3.98 \\
& & & & 16.80
\end{array}\right]
$$

describes the whole system.

These five coil systems will be powered individually by five power supplies of the thyristor type. The function of the power supplies is to provide the necessary time variations of the HELIAS magnetic field:

to charge the coils with a ramp rate of $\leq 10 \mathrm{~A} / \mathrm{s}$ - rectifier mode,

to discharge them after the operation with a ramp rate of $\leq$

\section{$-10 \mathrm{~A} / \mathrm{s}$ - inverter mode}

to stabilize currents at a specified level (of up to 40kA) during the energy production (steady-state operation).

The necessary voltages for this operation:

$(\mathbf{U})=\mathrm{d} / \mathrm{dt}\left[\left(\mathbf{L}_{\text {Helias }}\right)(\mathbf{i})\right]+(\mathbf{R})(\mathbf{i})=\left(\mathbf{L}_{\text {Helias }}\right) \mathrm{d}(\mathbf{i}) / \mathrm{dt}+(\mathbf{R})(\mathbf{i})$

where:

$\mathbf{i}^{\mathbf{T}}=\left[\mathrm{i}_{1}, \mathrm{i}_{2}, \mathrm{i}_{3}, \mathrm{i}_{4}, \mathrm{i}_{5}\right]-$ vector of the current reference values

$\mathbf{U}^{\mathrm{T}}=\left[\mathrm{U}_{1}, \mathrm{U}_{2}, \mathrm{U}_{3}, \mathrm{U}_{4}, \mathrm{U}_{5}\right]$ - vector of the voltages

$\mathbf{R}=\operatorname{diag}\left(\mathrm{R}_{\mathrm{i}}\right), \mathrm{i}=1,2, \ldots 5-$ diagonal matrix of the resistances of the busbars.

For the power supply units twelve-pulse AC/DC converters will be selected. The rectifiers are to be fed by two converter transformers the primary winding of the one being in star connection and the other in delta connection. On the DC side the two six-pulse rectifier groups are connected in parallel via a current balancing choke. This ensures a 12-pulse reaction on the grid.

By controlling of the delay angle, the converter operation can be continuously varied from charge mode (rectifier) to discharge mode (inverter), while the current flows continuously in the same direction. The active power flow through these converters is reversible (two-quadrant operation).

All five coil systems will be powered direct from the medium voltage plant grid for auxiliary system (utility interface), which will be divided in two subsystems (Figure 5).

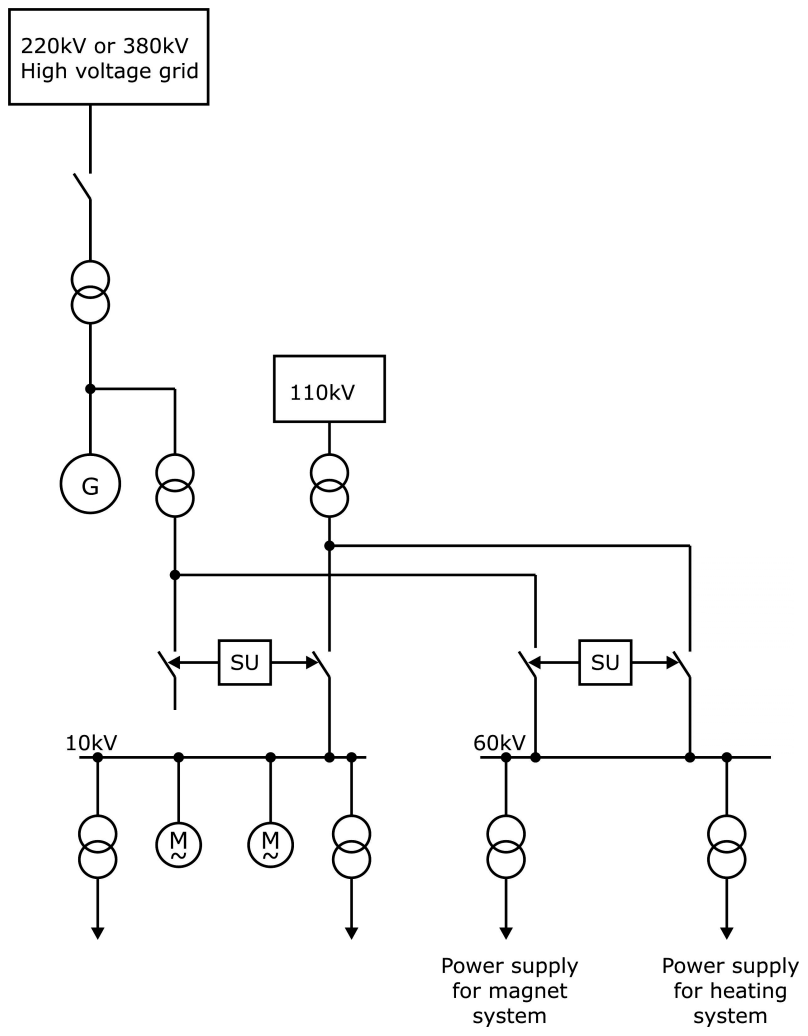


Fig. 5. The medium voltage utility interface for auxiliary system of the fusion power plant

One of them supplies all five coil systems and the plasma heating units and the second one the other loads in the plant with electric energy (Figure 6).

The grid is loaded by the operation of the line commutated converters with reactive power and harmonics. These harmonic currents flowing through the internal impedance of the utility source result in distorted voltage waveform at the point of common coupling. The voltage distortion at this point depends on the internal impedance of the ac source and the amplitudes of the injected harmonic currents. The reactive power consumption results in a very poor power factor of operation.

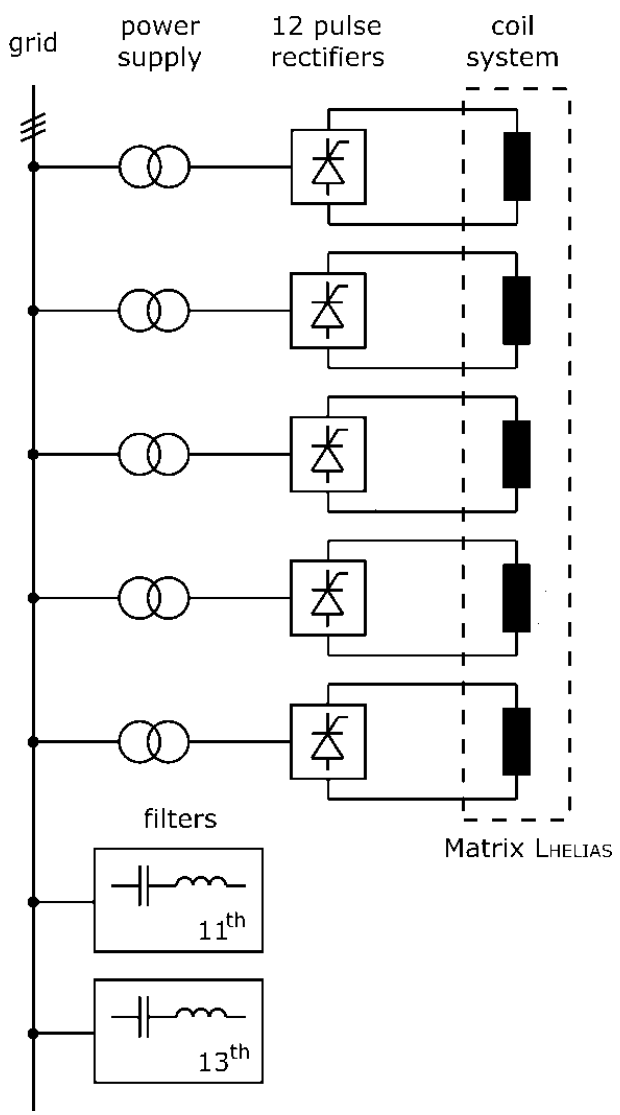

Fig. 6. Electrical scheme of HELIAS magnet system

The power-related parameters (power factor PF, representing the quotient of the active power and the apparent power, displacement factor DF, harmonic content, harmonic spectrum) are dependent upon loading, control and configuration of the converter and short-circuit levels of the grid. In addition to these problems, the phase-controlled converters cause notches in the voltage waveform of the utility system. If several converters are connected to the same supply mains, they will affect one another through the commutation notches. Operation directly in parallel is not possible, they must therefore be decoupled by transformer inductances [11].

The magnetic energy $\mathrm{W}_{\mathrm{m}}$ stored in the coil system of
HELIAS is:

$$
\mathrm{W}_{\mathrm{m}}=\frac{1}{2} \mathbf{i}^{\mathrm{T}} \mathbf{L}_{\text {HELIAS }} \mathbf{i},
$$

where $\mathbf{i}^{\mathrm{T}}=\left[\mathrm{i}_{1}, \mathrm{i}_{2}, \mathrm{i}_{3}, \mathbf{i}_{4}, \mathrm{i}_{5}\right]$ - is the 5-dimensional vector of the coil currents. With the operational currents of $40 \mathrm{kA}$ to achieve a magnetic field on axis of $5 \mathrm{~T}$ it amounts to $100 \mathrm{GJ}$.

A safety system, inserted between each power supply unit and the coil group, protects the superconducting coils in case of faults in the coils or in the power supply units [12].

\section{SIMULATION AND OPTIMIZATION OF THE SUPPLY UNITS}

The goal of the investigation is to optimize the magnet power supplies with respect to losses in components and interaction with the utility grid (low reactive power consumption from the grid, high power factor, low level of harmonics).

Investigation of the behavior of the equivalent electric schemes with complete models of the thyristor power supplies for the machine circuits were conducted with the SIMPLORER code [7]. This tool can be used to simulate complex power electronics systems including controls, on PCs operating under Windows.

One of the problems arising when designing cryogenic devices is the investigation of the magnetic field diffusion through the walls of the structural elements, i.e. in the casings of the coils, cryostats walls, vacuum vessel, radiation shields, etc. The accurate calculations of the losses of eddy currents, induced in the walls, are required for dimensioning of the cooling system.

The influence of the casing on the operation of the power supply system was investigated. Other passive elements like vacuum vessel, shields, etc, were not taken into account. By means of the Finite Element Network (FEN) Method the computation of diffusing magnetic fields and of the eddy currents induced during the transient process in the walls of the coil housing (Fig. 2) is converted to analysis of transient processes in electric networks [8]. The basic principle of the method is to represent a conducting surface, as a network comprised of a number of branches. The branches are properly interconnected and magnetically coupled to all external and internal circuit elements. Each branch has a resistance and a self-inductance as well as mutuals to all other branches. The matrix of the inductances was computed by an EFFI-code [9]. This code uses the analytical expressions and calculates the magnetic field, the magnetic forces and the inductances in coil systems for arbitrary geometry, where the non-planar coils are modelled by straight segments, called General Current Element (GCE). The accuracy of the computations depends on the chosen size of the GCE's and the numerical procedure. EFFI terminates the numerical evaluation when the relative difference between two successive iterations in the procedure is less than $10^{-5}$. The main inaccuracies occur by modelling the non-planar elements by straight segments. These elements adjoin at the top and bottom with small overlappings and gaps. An appropriate subdivision, depending on the local curvature 
of the coils, increases the accuracy of the results. The accuracy of such computations are in the order of $1 \%$. Eddy currents are then found by using a standard method for solving differential equations.

The SIMPLORER-model of one coil with a housing is shown in Figure 7.

a)

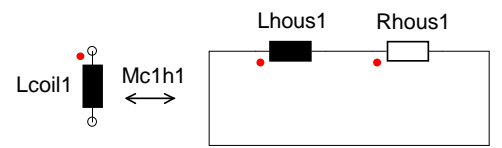

b)

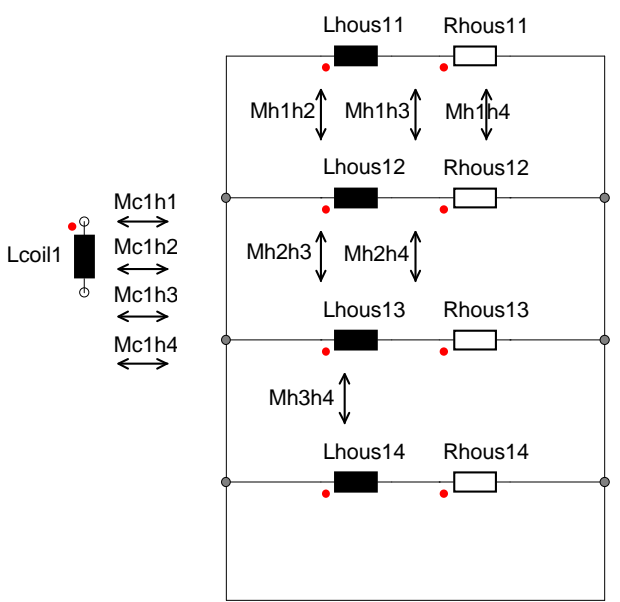

Fig.7. FEN-model of one coil with housing [11]

a)First approximation

b)Second approximation
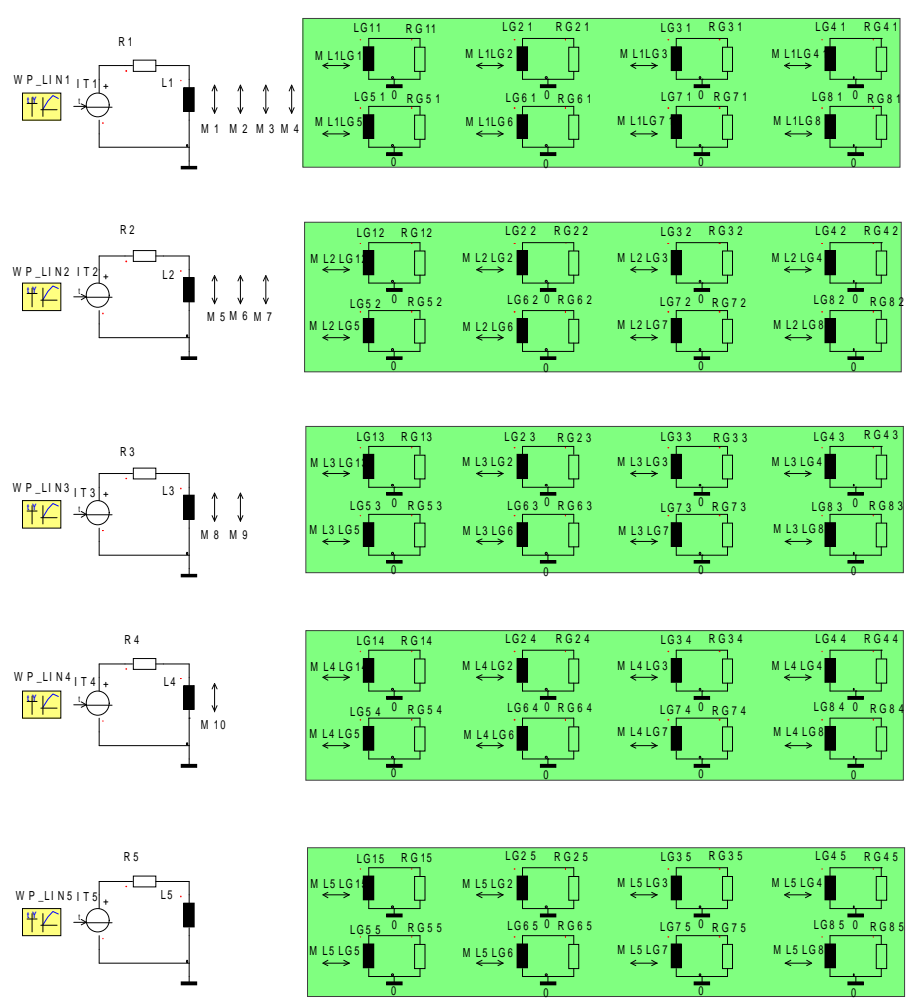

Fig. 8. The power supply system for HELIAS with converters represented by controlled current sources.

Many different classes of confinement configurations were studied, first with a simplified model of the power supply in which the converters were represented by controlled current sources ( Fig. 8).

To calculate the eddy-current losses in structural elements of the cryogenic devices during the magnetic field diffusion process exactly, the approach based on coupled solution of the electromagnetic and thermal problems must be applied, taking into account the strong dependence of resistivity, heat capacity and heat-transfer coefficient on temperature [10].

The results of one simulation with the maximum current values of $40 \mathrm{kA}$ in all coils are shown in fig. 9.

The necessary parameters of the power supplies are:

- nominal current $40 \mathrm{kA}$,

- $\quad 350 \mathrm{~V}$ no load voltage.

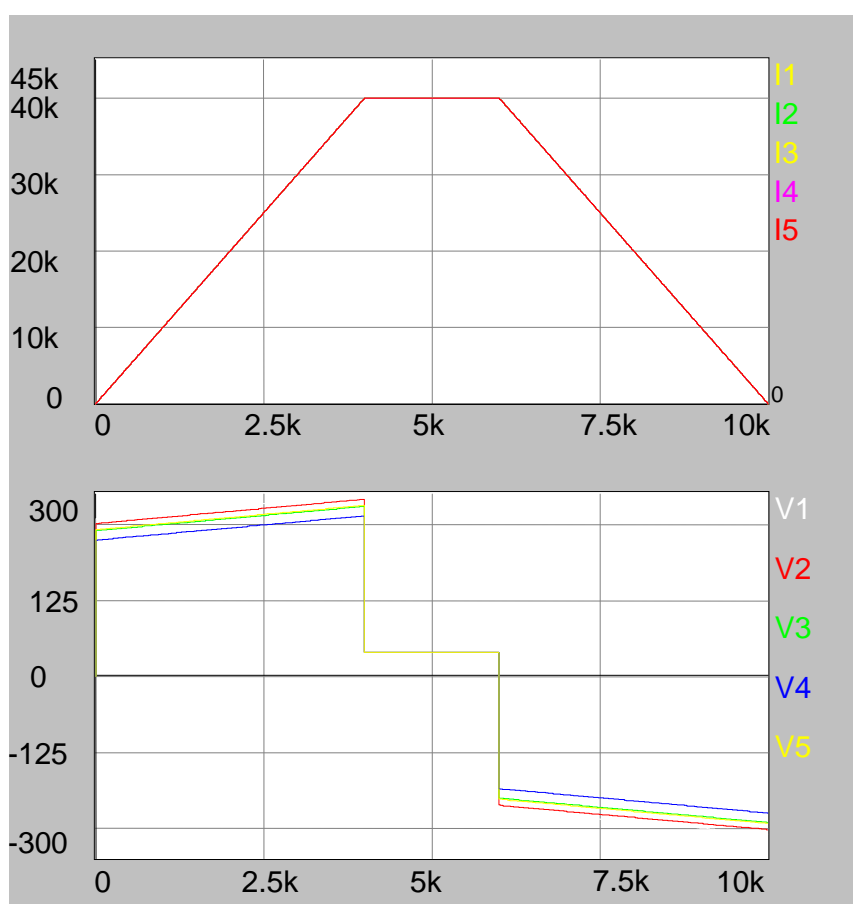

Fig. 9. Currents and voltages in the power supply for HELIAS with a simple model of the thyristor converter

A complete model of the thyristor power supplies and the housings was used to carry out the simulation of the dynamic behaviour of the system described by the L HELIAS-Matrix (Figure 10). 


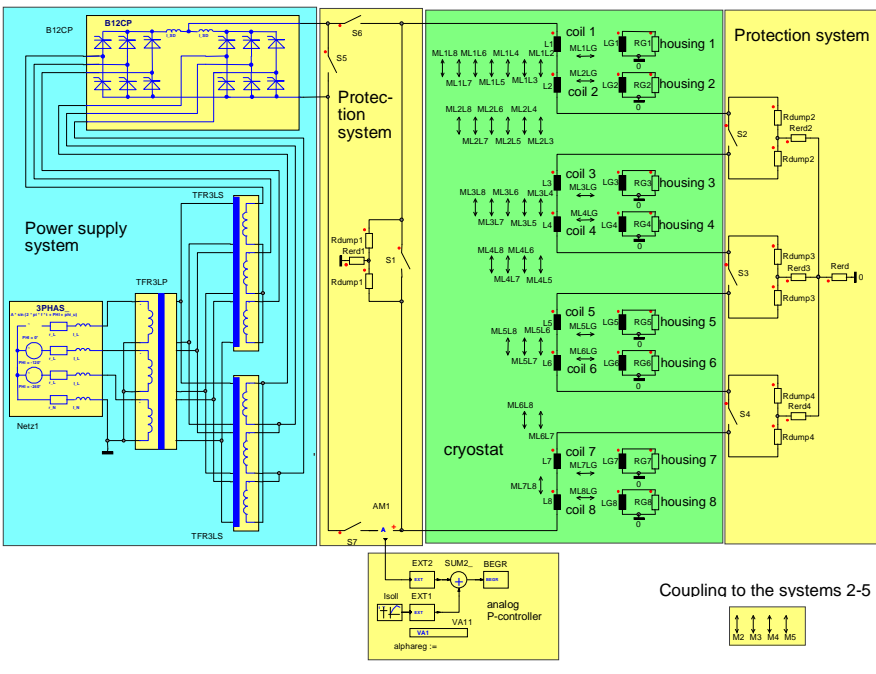

Fig. 10. SIMPLORER model of power supply and protection system for one coil system of HELIAS [11].

Figure 11 shows some exemplary results in one system during the operation. It illustrates the feasibility of stable coilcurrent control with 2-quadrant phase-controlled thyristor converters in HELIAS reactor in spite of magnetically coupling of the system.

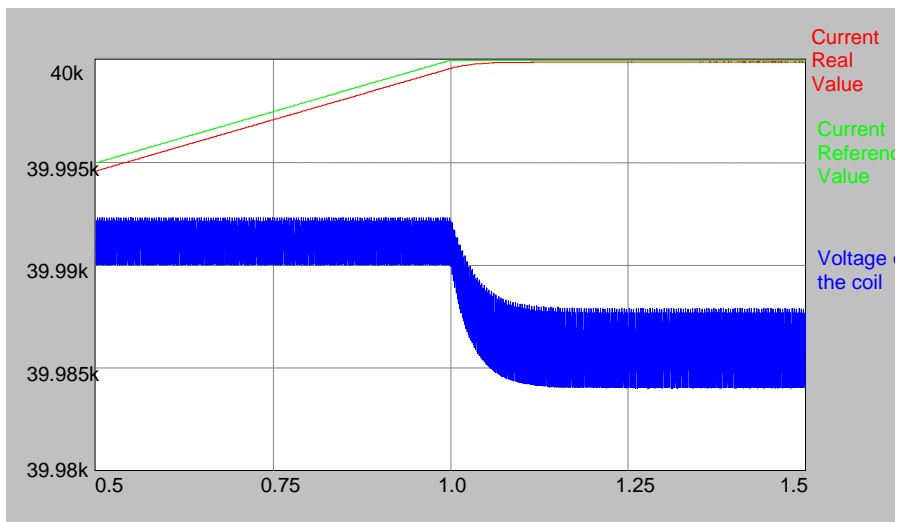

Fig. 11. Currents and voltages on the d.c. side in the power supply with a complete model of the converter

The thyristors control pulses (pulse timing) were synchronized with the supply voltage on the primary winding of the transformer. The reason to do this is the large commutation voltage drop on the secondary windings of the transformer (Figure 12), which causes big notches and might lead to a wrong voltage zero crossing detection.

To evaluate the extent of the distortions, the interaction of the harmonic currents with the AC grid was investigated by numerical network analysis with five modules connected to the bus. One result for the Flat-Top-Phase of operation is shown in figure 13. For the five twelve-pulse rectifiers system the phase angle is in the order of $85^{\circ}$, and $\mathrm{PF}$ is in the order of 0.1 , and must be improved to achieve an overall load power factor as close to unity as possible. The higher the power factor, the better is the utilization of the energy source.

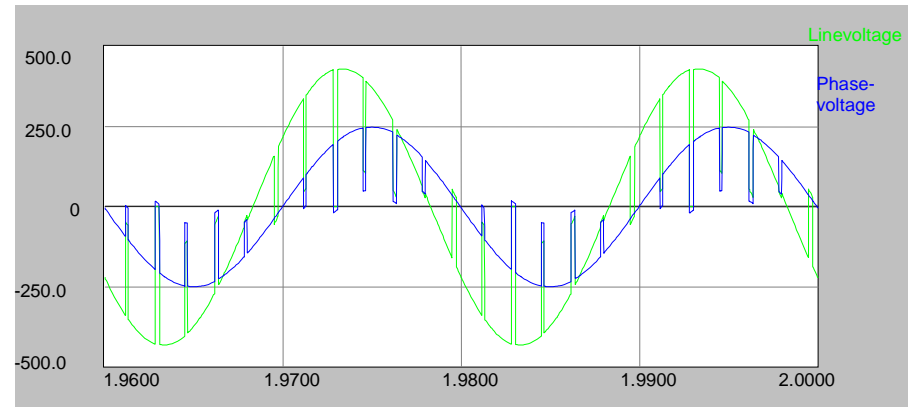

Fig. 12. Voltages on the secondary side of the transformer [11]

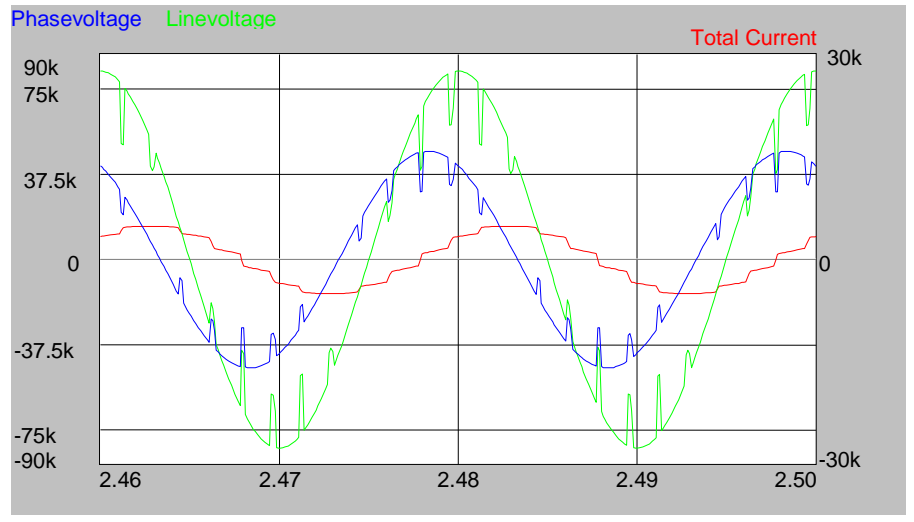

Fig. 13. Current, phase- and line voltages at the medium voltage utility interface [11]

The goal of optimization was to develop designs to reduce network pollution, increase the power factor and reduce the losses.

Because of high power levels associated with this application, it is important to reduce the harmonic currents generated on the ac side of the converter. This is accomplished by means of a 12-pulse converter operation. The twelve-pulse bridge generates only line current harmonics of the order $\mathrm{n}=$ $12 \mathrm{k} \pm 1$ (where $\mathrm{k}=$ an integer). The amplitudes of the harmonics are inversely proportional to their harmonic order and the lowest order harmonics are the eleventh and the thirteenth. These can be filtered out by tuned LC seriesresonant circuits connected in parallel with the converters. One filter unit consists of a bank tuned to the 11th harmonic and a damped highpass filter bank which reduces the 13 th and higher harmonics. These filters are capacitive at fundamental frequency and simultaneously they provide a large percentage of the reactive power for this frequency consumed by the converters.

An additional method to reduce the reactive power demand is changing the voltage under load with OLTCs (On Load Tape Changer) at the primary of the transformer [13]). The combination of OLTC and thyristor rectifier allows continuous regulation over the full range of application.

Simulation with SIMPLORER of the delay angle for the improved configuration is shown figure 14 . 


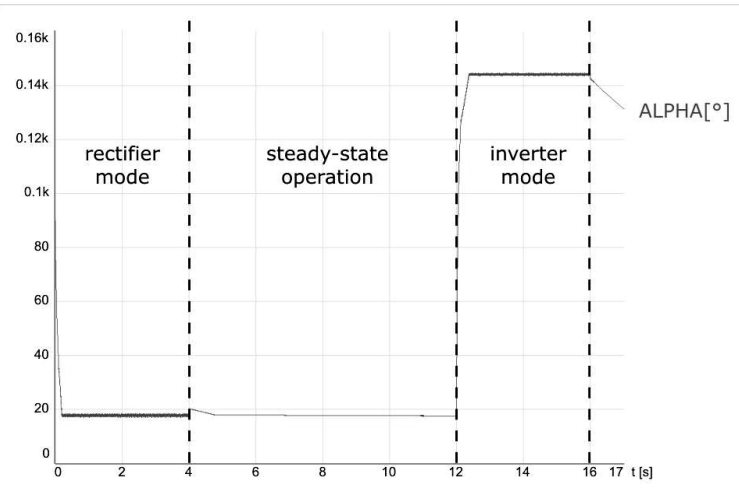

Fig. 14. Delay angle of the rectifiers during magnet operation

OLTC at the primary of the transformers and a filter system connected to the medium voltage grid help to significantly improve its power factor and thereby reduce the power line losses.

In the current flat-top operation the lagging reactive power demand of the converter can be adjusted with a help of OLTC to a leading reactive power of the filter banks, resulting in approximatevely unity power factor. The speed of this var regulation is determined by switching time of OLTC and is relatively slow (order of magnitude: seconds).

In the charging (current ramp-up) and discharging (current ramp-down) operation full dc-voltage is required: Therefore, the reactive power demand of the converter during ramp-up and ramp-down can not be controlled by OLTC. It is proportional to the dc-current and increases for instance from $0 \%$ to approx. $65 \%$ of the converter ideal dc-power $\mathrm{P}_{\mathrm{di} 0}$ during ramp-up from zero to the nominal dc-current. (Fig. 15).

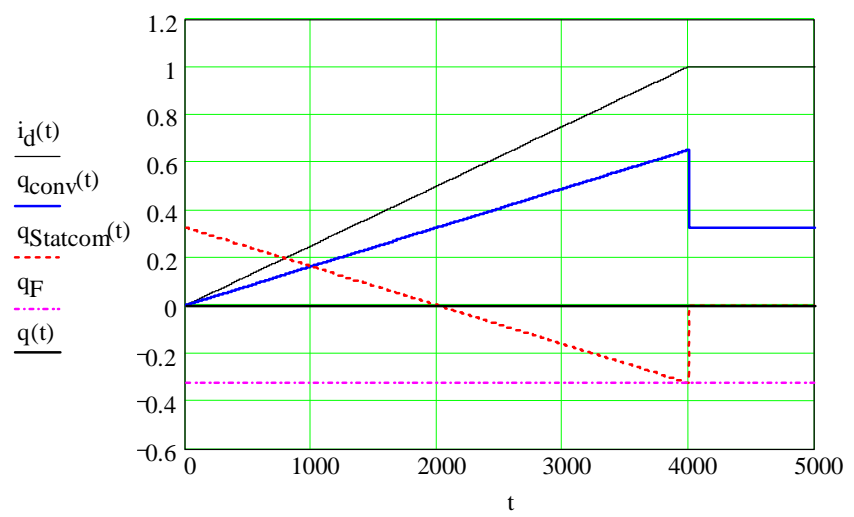

Fig. 15. Reactive power of Converter, Statcom, Filter banks during current ramp-up and flat-top Scale y-axis: p.u. , basis for reactive power : $\mathrm{P}_{\mathrm{d} i 0}$

Scale $\mathrm{x}$-axis: seconds

Improvement of the power factor in charging and discharging period, each of them taking more than 1 hour (4000s), can be achieved with Static Var Compensator (STATCOM) using Voltage Source Converter (VSC) topology. It shall be connected to the same bus as filter banks. In figure 15 STATCOM reactive power output in ramp-up and flat-top period is shown.

Due to its high dynamics STATCOM can compensate sudden reactive power change, which can not be followed by OLTC, and mitigate voltage fluctuations. STATCOM generates leading as well as lagging reactive power. Therefore, filter banks used for power correction can be basically reduced to a half capacity. Additionally, expecting further development in switching frequency of high-current semiconductor switch (e.g. IGCT), STATCOM can be used for active filtering of low order harmonics, e.g. 11th. Thus, the number of passive filter circuits can be reduced.

Converters using conventional network-commutated thyristors are still the best option regarding costs for controlled high dc-current supply. The basic drawback of such converter is its reactive power demand, especially for large delay firing angles, and high current harmonics requiring extensive filter circuits. Converters using turn-off semiconductors offer much better performance regarding reactive power control and harmonics. The most popular topology of self-commutated converters is VSC due to its simple structure, high efficiency and easy control. However, VSC can not be used directly for dc-supply of superconducting magnetic coils because of small allowable variation of dc-voltage. In order to control the current dc-chopper would be required and this makes the topology unattractive.

The dual circuit of the VCS is the Current Source Converter (CSC). The dc-link for CSC is a current source, as opposed to the voltage source in the VSC. Thus, CSC topology allows variation of dc-voltage including change of its polarity. CSC has in comparison to VSC the disadvantage of higher conduction loss and more complicated control, but the advantages, like easier overcurrent protection in case of shootthrough failure, let CSC topology to be discussed in recent time as a competitive candidate for high power applications [14]. Anticipating further development in semiconductor and converter technology, CSC multilevel topology [15] should be in future a series alternative to the conventional turn-on thyristor based converter for supplying high-current superconducting magnetic coils due to its much better performance regarding power factor and harmonics.

To improve the efficiency in view of low losses in the components it is necessary to use the $\mathrm{SiC}$ technology for the rectifiers [16].

\section{SIMULATION AND OPTIMIZATION OF THE SUPPLY UNITS}

The goal of the investigation is to minimize the grid loading during the start-up operation of the fusion reactor. The behavior of the power supplies were conducted with the NEPLAN code [7].

The ignition capability of the HELIAS has been investigated by calculations with different codes (ASTRA, POPCON) [2-4]. The minimum external heating power which leads to plasma ignition was found to be on the order of 50 MW, this coincides with the results of the other analysis where a heating power in the range from $60 \mathrm{MW}$ to $80 \mathrm{MW}$ were 
found. Due to the efficiency of the plasma heating system (gyrotron, quasioptical transmission line) in the order of 40$50 \%$, we need additional $160 \mathrm{MW}$ active power from the grid during the heat-up phase of $15 \mathrm{~s}$.

The investigation covers two periods: the start-up operation and the energy generation stage of the fusion power plant. At the start-up operation the total load (the magnetic confinement system, the plasma heating system, the cooling system etc.) is powered from the grid. During the energy generation of the plant, the plasma heating is turned off, and the load is powered from the generator.

The following figure demonstrates the start-up operation of the power plant without the energy storage unit simulated with the NEPLAN-Code (Figure 4).

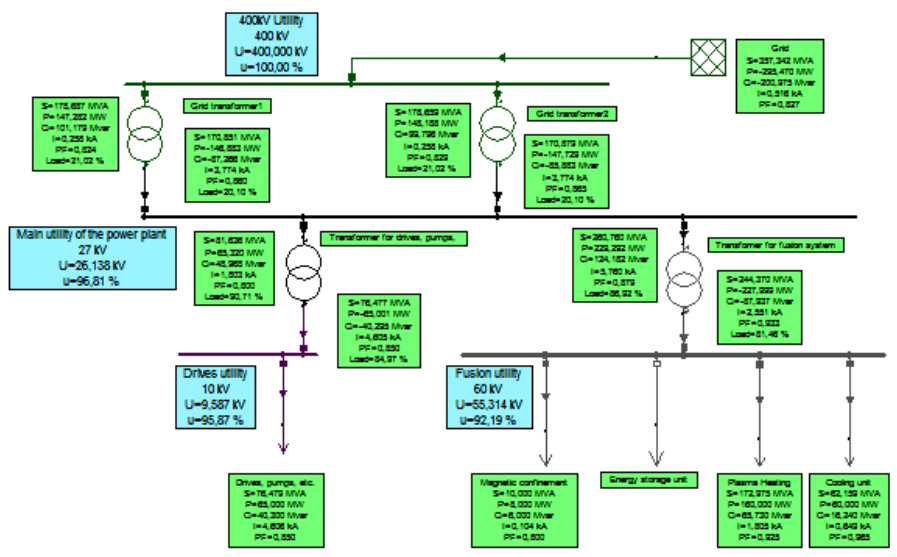

Fig. 16 Start-up operation of the fusion power plant without energy storage system [14].

The new approach is to use an additional energy storage unit to reduce the network loading (Figure 5).

For such case it can be used:

- the optimized magnetic confinement system (superconducting magnet system, multiconverter unit and STATCOM) as a SMES or

- modular flywheel storage unit in the startup operation of the power plant [15].

In this paper the second possibility will be presented.

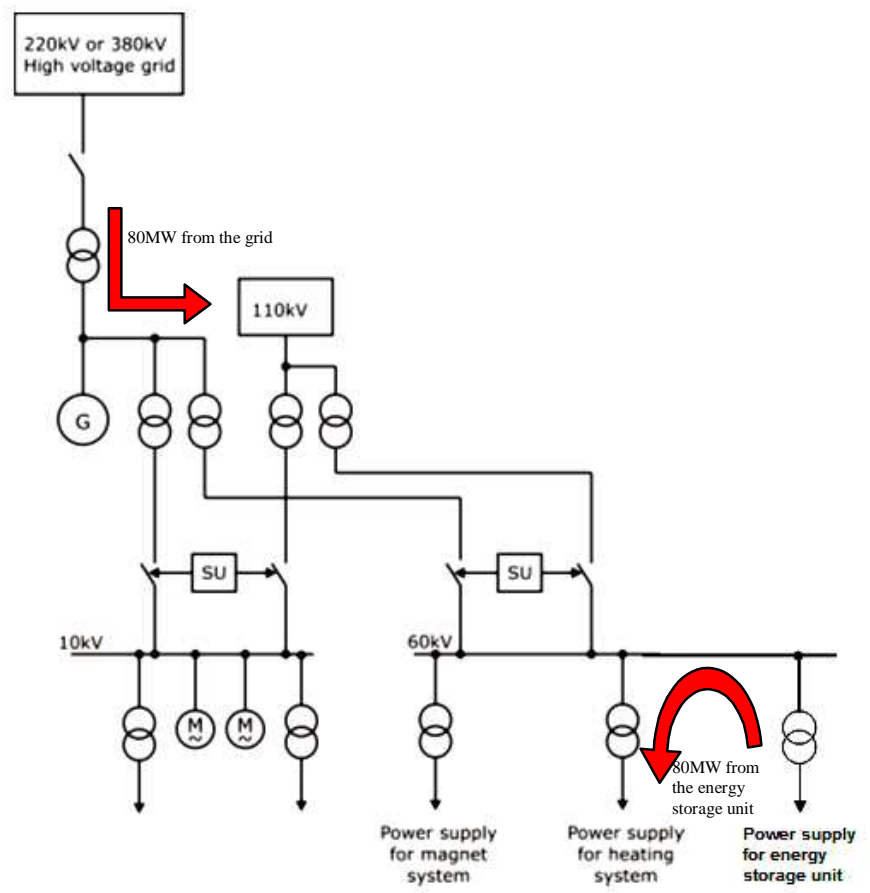

Fig. 17 Advanced medium voltage utility interface for auxiliary system of the fusion power plant during plasma heat-up

The Magnetodynamic Storage (MDS) from the company Magnet Motor in Starnberg/Germany (figure 6) [8] is a flywheel storage unit with a vertical rotation axis. The rotor is a hollow cylinder primarily made of carbon fiber composite. For an optimum compact system design the motor/generator $(\mathrm{M} / \mathrm{G})$ unit is integrated inside the hollow rotor.

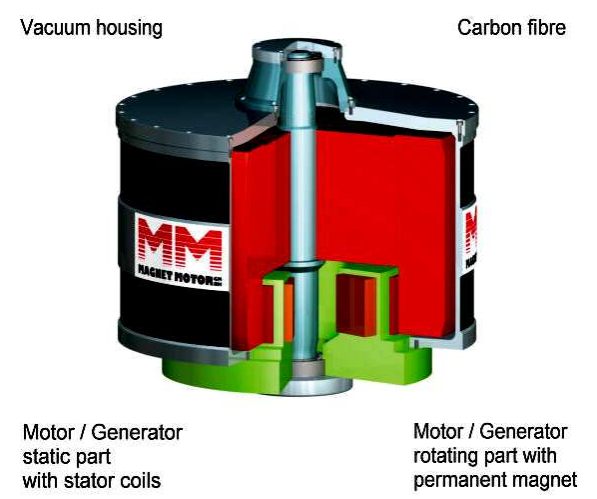

Fig. 18 Magnetodynamic Storage (MDS)[8].

The MDS gains stored energy while the M/G unit is run as a motor, accelerating the rotor. The MDS feeds back energy when the $\mathrm{M} / \mathrm{G}$ unit is switched to generator mode, thus reducing the rotor speed. In order to minimize bearing friction, most of the rotor weight is borne by magnetic forces. The MDS housing is evacuated, thus minimizing air friction losses.

The connection of two units to the grid is shown in figure 7. Ten such groups are necessary for an 80MW, 1.2GJ energy storage unit. 


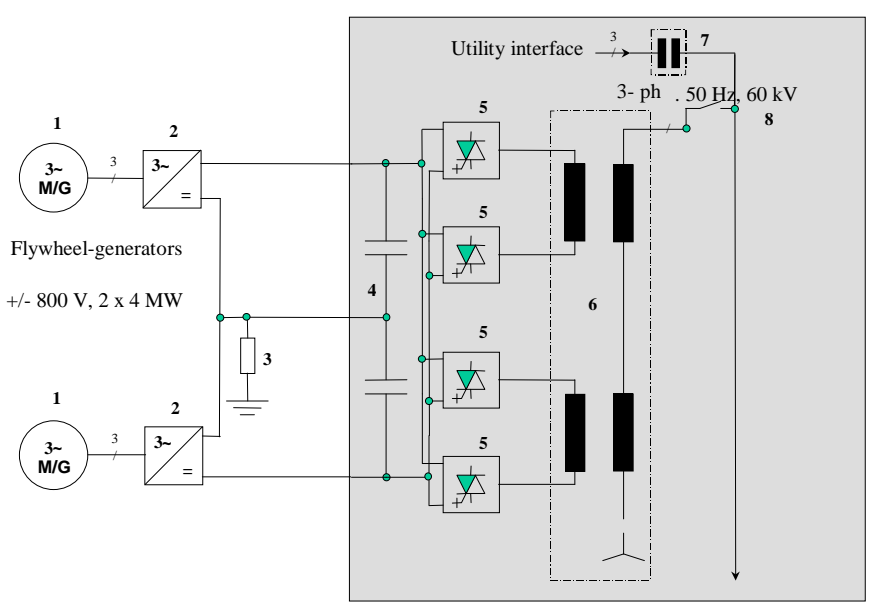

Fig 19. Grid connection of two 4 MW MDS with IGCT-Inverters [15]

The results of the simulation for the auxiliary system with the energy storage unit are shown in Figure 8. In compare to the figure 4 the loading of the grid is much smaller during the start-up operation. The active power pulsed loads cause a power frequency changes in the grid which oscillate. These oscillations produce transient power flows which can danger the overall grid stability.

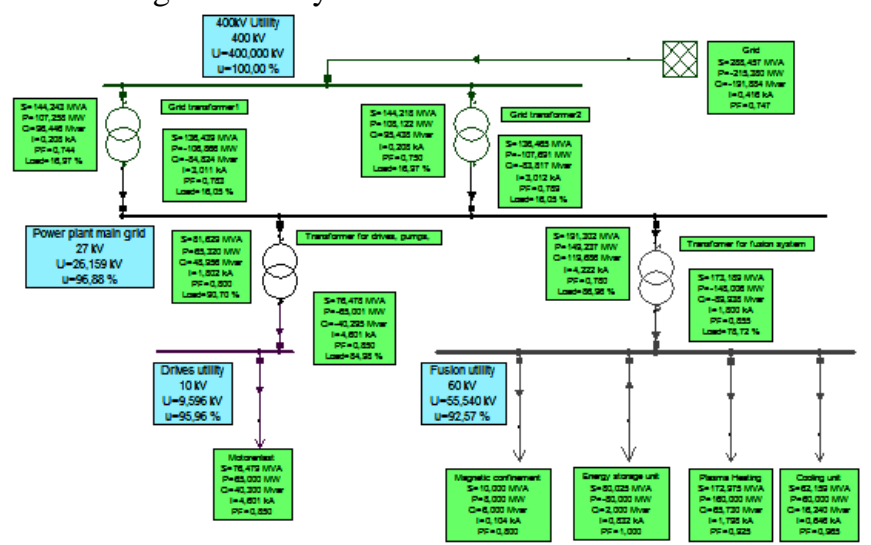

Fig. 20 Start-up operation of the fusion power plant with $80 \mathrm{MW}, 1.2 \mathrm{GJ}$ energy storage system [14]

The impact of the pulse load $\mathrm{P}_{\mathrm{L}}$ can be approximated using the formula:

$$
\Delta \mathrm{f}=\frac{P_{L}}{E_{R}}
$$

where $\Delta \mathrm{f}$ is the frequency change and $\mathrm{E}_{\mathrm{R}}$ is the network regulating energy (network stiffness) [12]. The European interconnected grid has $\mathrm{E}_{\mathrm{R}}=18 \mathrm{GW} / \mathrm{Hz}$; a $250 \mathrm{MW}$ absorption causes a frequency variations of $14 \mathrm{mHz}$; similar to the background frequency variations $(20 \mathrm{mHz})$.

\section{CONCLUSIONS}

A power supply system for feeding the superconducting coils of Helias fusion reactor has been investigated and optimized. The goal was to achieve a small negative impact to the power grid and low losses in the components. The design of the optimized multiconverter supply system has been studied by means of computer simulations, using the SIMPLORER code and EFFI code as a pre-processor. The approximation with the Finite Element Network (FEN) method allows the investigation of the whole coil system including power supplies and passive structures.

The combined application of the 12-pulse thyristor rectifier, the STATCOM compensator and a filter system connected to the medium voltage grid results in a significant improvement of the power factor and thereby reduction of the power line.

A power supply system for feeding the auxiliary system of HELIAS fusion reactor has been investigated and optimized.

The goal was to reduce network loadings in the power plant start-up phase during plasma heating. The design of the supply system has been studied by means of computer simulations, using the NEPLAN code.

The new approach is the use of an additional energy storage unit:

- the optimized magnetic confinement system (superconducting magnet system, multiconverter unit and STATCOM) as a SMES or

- the modular flywheel storage unit

in the startup operation of the power plant.

These applications offer a considerable reduction of network loading during the plasma heating phase.

\section{REFERENCES}

[1] Kernfusion - Stand und Perspektiven, Max-Planck-Institut für Plasmaphysik (IPP), Garching b.München, 3. Auflage, 1993.

[2] Kernfusion - Berichte aus der Forschung, Max-Planck-Institut für Plasmaphysik (IPP), Garching 1995.

[3] Wobig $\mathrm{H}$. et al, Recent Developments in Helias Reactor Studies, Proceedings of the 19th IAEA Fusion Energy Conference, Lyon, France, Oct. 14-19, 2002.

[4] Beidler C.D.et al, The Helias Reactor 4/18, Nuclear Fusion, Vol.41, No.12, (2001), 1759-1766

[5] Grieger, G., Nührenberg, J., Renner, H., Sapper, J., Wobig, H., HELIAS stellarator reactor studies and related European technology studies, Fusion Engineering and Design, Vol.25, (1994), 73.

[6] Harmeyer E., Kißlinger J., Wieczorek A., Wobig H., Superconducting Coil System for a Stellarator Fusion Reactor, MT-17, Geneva, Suisse, 2001, IEEE Transactions on Applied Superconductivity, Vol.12,No.1, (2002), 558-561.

[7] Simulation System SIMPLORER - user manual, Copyright 1996-2005 Ansoft Corporation

[8] A.Farschtschi, Dreidimensionale Wirbelstromberechnung nach der Netzwerkmethode, etzArchiv, Bd.8 (1986) H.5, 165-169.

[9] Sackett, S.J.: EFFI - A Code for Calculating the Electromagnetic Field, Force an Inductance in Coil Systems for Arbitrary Geometry, Lawrence Livermore National Laboratory, Report UCRL-52402 (1978)

[10] V.Sokolowsky, V.Meerovich, M.Slonim, Eddy current losses at cryogenic temperatures, IEEE Transations on Magnetics, Vol. 29, No. 3, May 1993, 2095-2098

[11] Simulation System SIMPLORER - user manual, Copyright 1996-2005 Ansoft Corporation

[12] NEPLAN Brochure Electricity

[13] http://www.magnet-motor.de/

[14] Wieczorek A., Harmeyer E., Linhofer G., Computer Simulations of the Power Supply for the Superconducting Coils of Helias Stellarator Fusion Reactor, Proceedings of EPE 2003: 10th European Conference on Power Electronics and Applications, Toulouse, France, Sep 2-4, 2003.

[15] Zhenxue $\mathrm{Xu}$, Advanced semiconductor Device and Topology for High Power Current Source Converter, Dissertation, Virginia Polytechnic Institute and State University, Dec. 2, 2003. 
[16] K.Imaie, O. Tsukamoto and Y. Nagai, Control strategies for multiple parallel current source converters of SMES system, IEEE Transactions on Power electronics, Vol. 15, March 2000, pp. 377-385.

[17] Rostagni,G., The electric power handling from present machines to fusion power stations, Fusion Engineering and Design, Volume 74, issues 1-4, November 2005, pp. 87-95

[18] O.Gaupp, E.Harmeyer, A.Wieczorek, H.Wobig, Power Supply Optimization for the Superconducting Coil System of HELIAS Fusion Reactor, IEEE ISIE2006 Montreal, 9-13 July 2006, Canada.

[19] C.Buchner, Untersuchung des elektrischen Eigenbedarfs für Fusionskraftwerke mit NEPLAN, Diploma work, Regensburg University of Applied Sciences, Regensburg, 2006.

[20] Wieczorek, A., Energiequelle 40MW für 15s. 600MJ, Untersuchung und Bericht für das Max-Planck-Institut für Plasmaphysik (IPP), Garching, 2001.

[21] Wieczorek A., Harmeyer E., Linhofer G., Simulation Studies of the Protection System for the Superconducting Coils of Helias Stellarator Fusion Reactors, Proceedings of the 20th IEEE/NPSS Symposium on Fusion Engineering 2003, San Diego, CA, Oct 14-17, 2003.

[22] Axel Krämer, On-Load Tap-Changers for Power Transformers,

Regensburg, 2000 Maschinenfabrik Reinhausen GmbH.

[23] Zhenxue Xu, Advanced semiconductor Device and Topology for High Power Current Source Converter, Dissertation, Virginia Polytechnic Institute and State University, Dec. 2, 2003.

[24] K.Imaie, O. Tsukamoto and Y. Nagai, Control strategies for multiple parallel current source converters of SMES system, IEEE Transactions on Power electronics, Vol. 15, March 2000, pp. 377-385.

[25] T.Matsukawa, R.Shimada, Efficiency Improvement of AC/DC Converter Using SiC-based Power Electronics Device, Proceedings of the 20th IEEE/NPSS Symposium on Fusion Engineering 2003 , San Diego, CA, Oct 14-17, 2003.

(M'76-SM'81-F'87) and the other authors may include biographies at the end of regular papers. Biographies are often not included in conferencerelated papers. This author became a Member (M) of IEEE in 1976, a Senior Member (SM) in 1981, and a Fellow (F) in 1987. The first paragraph may contain a place and/or date of birth (list place, then date). Next, the author's educational background is listed. The degrees should be listed with type of degree in what field, which institution, city, state or country, and year degree was earned. The author's major field of study should be lower-cased.

The second paragraph uses the pronoun of the person (he or she) and not the author's last name. It lists military and work experience, including summer and fellowship jobs. Job titles are capitalized. The current job must have a location; previous positions may be listed without one. Information concerning previous publications may be included. Try not to list more than three books or published articles. The format for listing publishers of a book within the biography is: title of book (city, state: publisher name, year) similar to a reference. Current and previous research interests ends the paragraph.

The third paragraph begins with the author's title and last name (e.g., Dr. Smith, Prof. Jones, Mr. Kajor, Ms. Hunter). List any memberships in professional societies other than the IEEE. Finally, list any awards and work for IEEE committees and publications. If a photograph is provided, the biography will be indented around it. The photograph is placed at the top left of the biography. Personal hobbies will be deleted from the biography.

\section{O.Gaupp}

R.Haller

E.Harmeyer

J.Mühlbacher

A.Wieczorek

H.Wobig 